\title{
Measurements of a single pulse impinging jet. A CFD reference
}

\author{
Mirko Bovo ${ }^{1,2, \mathrm{a}}$, Borja Rojo ${ }^{1}$ and Maxim Golubev ${ }^{1}$ \\ ${ }^{1}$ Chalmers University of Technology, Applied Mechanics, Dep. Thermo-Fluid Mechanics, Göteborg, Sweden \\ ${ }^{2}$ Volvo Car Corporation, Powertrain, Göteborg, Sweden
}

\begin{abstract}
This paper reports three sets of measurements of a single pulse impinging jet. The purpose is to serve as a reference for CFD validation. A gas injector generates a single pulse jet at Re $\sim 90000$. The jet impinges on a temperature controlled flat target at different angles $\left(0^{\circ}, 30^{\circ}, 45^{\circ}\right.$ and $\left.60^{\circ}\right)$. The jet velocity field is measured with PIV. The evolution of the jet velocity profile in time is reported at two different locations (suitable as CFD inlet conditions). At the same locations also turbulence quantities are reported. The impingement wall temperature is measured with fast responding thermocouples and infrared camera. These give high time and space resolution respectively. Results are reported in a format suitable for comparison with CFD simulations. The results show that the heat transfer effects are highest for the jet impinging normally on the target. Target inclination has remarkable effects on the jet penetration rate and repeatability. Even small target inclinations result creates a preferential direction for the jet flow and cause a shift in the position of the stagnation region.
\end{abstract}

\section{Introduction}

Jets are interesting flows found in many natural events and industrial applications. Good reviews of imping gas jet are $[1,2]$, the second extending the work also to flame jets. Impinging jets are known for their high levels of heat transfer, this characteristic is of great interest. They have been studied in a number of configurations varying in geometrical and dynamic parameters. The historic reference for impinging jet heat transfer is [3] and, for the flow field, [4]. These works were the first to report measurements of the impinging jet in a format well suited for comparison and validation of CFD (Computational Fluid Dynamics) models. Since then, a number of experimental investigation using different techniques and jet configurations were carried out, e.g. $[5$ - 11]. Impinging jets have particular thermo-fluid characteristics which make them difficult to model and are indeed a recommended test case for turbulence models [3]. For these reasons they are focus of much research.

In many cases jets are not stationary (i.e. continuous) but are instead delivered as a pulse. Works on pulsating impinging jet are found with relation to cooling of electronic components. In these cases the material found is characterized by multiple consecutive pulsations where the each pulse is partially affected by the previous one [14 - 17].

In some cases the jet pulse is completely independent of the previous ones (single pulse jet). An example of single pulse impinging jet is the fuel injection in diesel engines. At every power stroke a spray of fuel is injected into the combustion chamber resulting in a jet-like pulse impinging on the wall (e.g. [12]). In most cases, the jet impinges on the piston surface with changing angles due to the piston's shape and motion. This event is the starting inspiration for this work (see also [13]).

The primary purpose of the experimental campaign is to collect data on single pulse jet impingement for a meaningful comparison with CFD simulation. The experiment is designed so that an interested researcher can replicate the case in the numerical environment. The flow is measured with various techniques to capture the jet velocity field and its thermal effects on the impingement wall. The flow field is measured with PIV (Particle Image Velocimetry). A set of measurements is carried out to record the jet velocity profile at locations suitable as CFD boundary conditions. These are also further elaborate to extract some information about the turbulence levels in the flow. Other PIV measurements focus instead on the entire jet space-time evolution and are reported in [13]. The jet-wall thermal interaction is measured with two distinct experiments using fast responding thermocouples and infrared thermal camera. These different techniques allow resolving the event with high resolution in time and space respectively. The jet thermal effect on the impingement target is also reported in space and time. The results format is chosen to be suitable for comparison with CFD results.

\footnotetext{
$\overline{{ }^{a} \text { Corresponding bovo@chalmers.se }}$
} 


\section{The case, experimental setup}

The gas jet is generated with a modified injector for gasoline DI engines, the design is inspired by [18]. The original injector tip/nozzle is cut off exposing the needle (see figure 1). A single hole nozzle is machined from a brass rod and threaded to the injector stem. To ensure good repeatable sealing the injector is run for some time previous the experiment. This way the hard steel needle hammers a good seat on the more ductile brass nozzle.

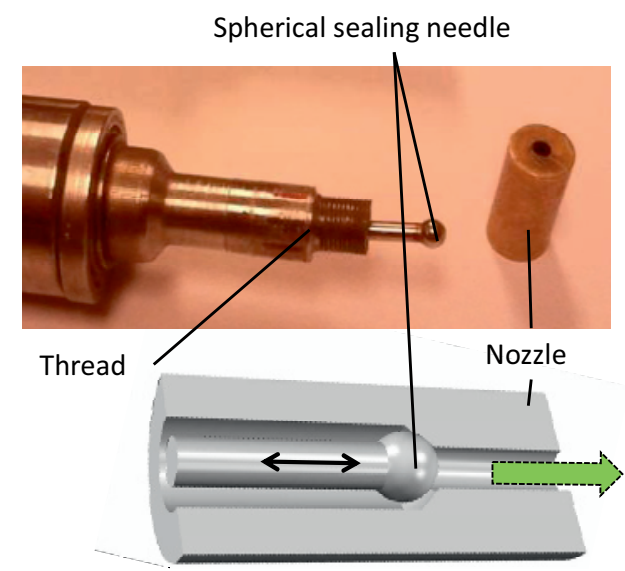

Figure 1. Modified GDI injector and nozzle assembly.

The nozzle is a $1 \mathrm{~mm}$ diameter, $6 \mathrm{~mm}$ long hole. The fluid used is synthetic air. The conditions upstream the needle are 10 bar and $20{ }^{\circ} \mathrm{C}$. The jet is discharged in quiescent air at atmospheric conditions generating an under-expanded jet. A description of this type of flow can be found in [18]. The flow is supersonic at the nozzle but rapidly becomes subsonic through a series of expansion shocks. This study focuses on the region downstream the transition region. The jet is directed to a temperature controlled surface at $150{ }^{\circ} \mathrm{C}$. The target is a circular aluminum plate, in its core are fitted two $600 \mathrm{~W}$ electrical resistances. Handbook calculations show that such element affected by natural convection has practically a uniform temperature (i.e. well within the accuracy of the measurements). The target temperature is controlled by a closed loop PDI controller monitoring the signal of a thermocouple opportunely placed. The target is positioned at $40 \mathrm{~mm}$ from the nozzle and is tilted as shown in figure 2 .

The injection duration is $5 \mathrm{~ms}$ and the jet is fully developed after $\sim 1 \mathrm{~ms}$ from start of injection. A reference Reynolds number ( $\mathrm{Re}$ ) of $\sim 90000$ is associated to the fully developed jet. $\operatorname{Re}$ is calculated upon the approximate width of the jet and the jet peak velocity 20 $\mathrm{mm}$ from the impingement wall. From start of injection the jet takes about $0.75 \mathrm{~ms}$ to reach the target with an average velocity of $40 \mathrm{~m} / \mathrm{s}$. The sudden expansion at the nozzle causes a significant temperature drop. Handbook thermodynamic calculation predicts a temperature decrease of $\sim 58 \mathrm{~K}$ at the nozzle exit. Travelling downstream the jet entrains a considerable amount of air with an estimated temperature difference from the environment of $8.5 \mathrm{~K}$ at $20 \mathrm{~mm}$ from the nozzle. This estimate does not include viscous losses nor heat transfer effects, both of which tend to even out the temperature difference between the jet and its surrounding.

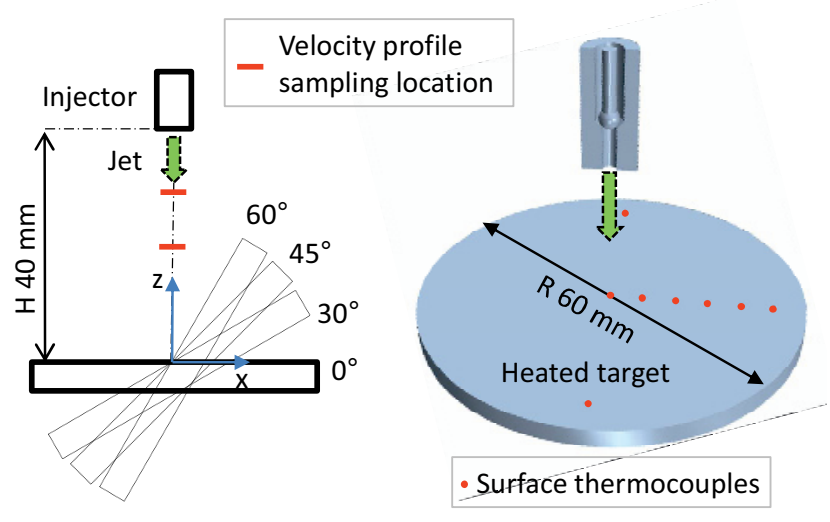

Figure 2. Target geometry and position relative to nozzle.

\section{PIV}

PIV is a well-established technique to measure flow velocity. General description and guidelines to perform PIV are found in [20]. In this work, the flow is investigated using the stereo-PIV system commercialized by LaVision. The PIV setup used in the experiments consists of an Imager ProX 4M camera (2048 x 2048 pix resolution), synchronizing device, $\mathrm{Nd}$ :YaG double-pulsed laser $(532 \mathrm{~nm})$. To align the measuring plane with the jet axis a hanging bob with $0.1 \mathrm{~mm}$ thread is attached to the nozzle. Using the camera live-mode the laser sheet is adjusted to strike the bob thread. The system spatial calibration (scaling) is performed using a ruler. The scaling accuracy does not exceed $1 \%$. The system is coupled to the injector opening signal giving the possibility to control the acquisition delay relative to injection start. The jet is not released in an enclosure but in ambient air which practically contains no particles. Previous to the PIV analysis, the recorded images are preprocessed by subtracting the sliding background of 8 pixel scale and applying particle intensity normalization (available in DaVis software). The velocity fields are extracted decreasing from $128 \times 128$ pix ( 2 passes) to 64 x 64 pix (3 passes). An interrogation window with $50 \%$ overlapping is used. Average variables are based on 100 samples. In supersonic and, partially, in transonic regions the velocity pulsations are quite large. Associated to this, the lack of particles and their mixing produces low quality correlation peaks and noises in the results. Nevertheless, the supersonic region is not in the scope of this work. Bad vectors are filtered out using postprocessing the routine extensively presented by DaVis. Correlation peak value filtration and $5 \times 5$ region outlier are used.

The flow is seeded with cigarette smoke. The smoke consists of a high concentration $80-100 \mathrm{~nm}$ particles. The particle size and density are appropriate to follow the jet flow in the subsonic region. The cigarette is located in a $\sim 1 \mathrm{dm}^{3}$ vessel fed with synthetic air. From the vessel a short line feeds the injector. The best concentration was found with the cigarette in series with the injector line. The cigarette filter is cut to a $1 / 5$ of the original length 
and a coarse metal mesh is inserted to prevent the cigarette to be pushed into the injector line. The PIV GUI allows to readily monitoring the seed concentration. The injector is cleaned every $5-10$ cigarettes flushing the system with acetone. Every cigarette allows for seeding of $\sim 1000$ injection (at $\sim 2 \mathrm{~Hz}$ ). This assembly results in a readily available, standardized and cheap solution for seeding high-pressure/low-mass-flow systems (figure 3).

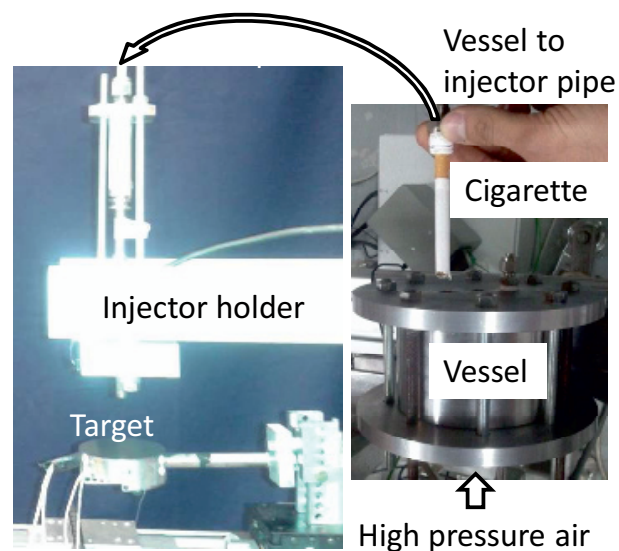

Figure 3. Seeding system.

\subsection{PIV results}

The measuring plane (the laser sheet) is arranged to pass through the nozzle axis and the target maximum inclination (see coordinate system in figure 2). Measurements are carried out at different time delays from start of injection to follow the jet development. The PIV system allows for a fine resolution of the instantaneous jet flow in space. An example of the instantaneous flow field is presented in figure 4.

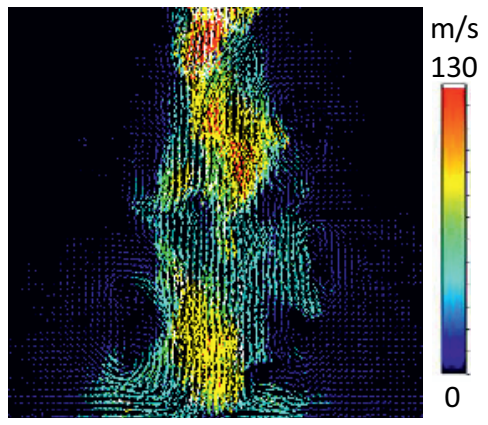

Figure 4. Example of PIV instanteneous results.

Collections of images presenting the evolution of the flow field are published in [13]. This work focuses on reporting results suitable as input to CFD simulation.

\subsection{Cross jet velocity profile}

The jet tip evolution is finely resolved to provide good space-time resolution of velocity profile. The velocity profiles are reported at $10 \mathrm{~mm}$ and $20 \mathrm{~mm}$ downstream the nozzle, these are the average of 100 samples. The interrogation window for this set of measurements covers an area of $\sim 20 \times 20 \mathrm{~mm}$ centered $\sim 15 \mathrm{~mm}$ from the nozzle tip. The system is capable to capture only one sample per jet, therefore it is not possible to follow space - time evolution of an individual event. In the initial part of the injection it is possible to follow the evolution of the jet tip penetration. This period is resolved with acquisitions every $0.01 \mathrm{~ms}$ until the jet establishes a developed state. This allows resolving the large vortexes at the jet tip. Increasingly longer time steps are used afterwards to record the entire jet duration. Figure 5 presents the early stages of the jet pulse. The tip of the jet is first detected $10 \mathrm{~mm}$ downstream the nozzle after $\sim 0.44 \mathrm{~ms}$ from the injector opening signal and it stabilizes at about $\sim 320 \mathrm{~m} / \mathrm{s}$. The radial component $(\mathrm{x})$ has the largest velocity variation in correspondence with the passage of the jet tip (time $\sim 0.44 \mathrm{~ms}$ ). The jet $\mathrm{x}$ velocity profile is rapidly stabilizing at values of $\sim 20 \mathrm{~m} / \mathrm{s}$ from time $\sim 0.46 \mathrm{~ms}$.

Unphysical results are visible at the margins of most figures, for example a $z$ velocity component of $-200 \mathrm{~m} / \mathrm{s}$ in position $-4 \mathrm{~mm}$ in figure 5 . The system is calibrated for velocity typical of the jet core $100-200 \mathrm{~m} / \mathrm{s}$. Any region with velocity much different than the calibration can be subjected by very large errors. Nonetheless, a reader with careful observation can with ease extract the meaningful part of the velocity profile. All above implies that the $x$ velocity component is also affected by a perceptually larger error compared to the $\mathrm{z}$ velocity component.
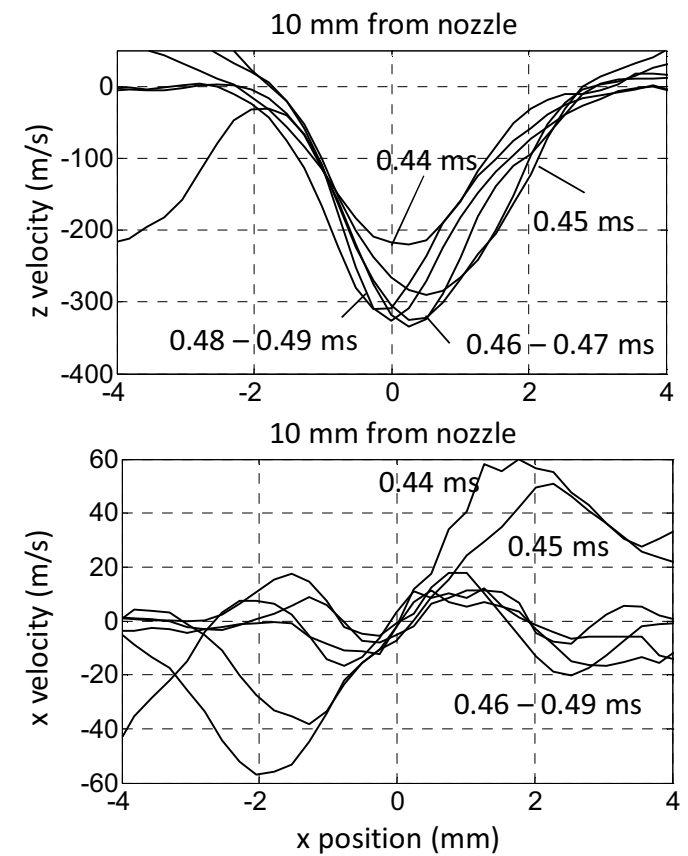

Figure 5. $\mathrm{z}$ and $\mathrm{x}$ velocity profiles $(0.44-0.49 \mathrm{~ms})$.

The jet tip is first detected $20 \mathrm{~mm}$ from the nozzle at time $0.50 \mathrm{~ms}$ (figure 6). A noticeable velocity fluctuation is detected for the same time $10 \mathrm{~mm}$ from the nozzle with no evident explanation. At the same time $(0.50 \mathrm{~ms})$ the largest fluctuation is also detected in the radial direction (figure 7), much like the passage of the jet tip described previously (at $10 \mathrm{~mm}, 0.44 \mathrm{~ms}$ ). After the tip passage, the jet quikly stabilizes at $\sim 230 \mathrm{~m} / \mathrm{s}$.

At time $0.58 \mathrm{~ms}$ a sudden reduction in velocity is recorded $10 \mathrm{~mm}$ from the nozzle and the jet changes 
regime (figure 8 , notice the $\mathrm{y}$-axis scale). The $\mathrm{z}$ velocity component stabilizes at $\sim 140 \mathrm{~m} / \mathrm{s}$ until end of injection.
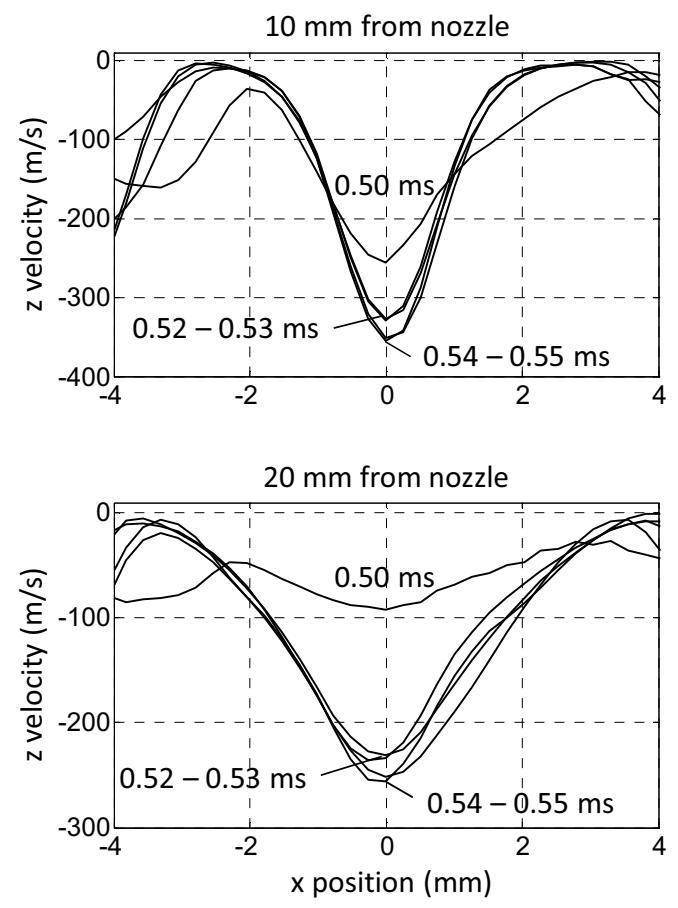

Figure 6. $\mathrm{z}$ velocity profiles $(0.50-0.55 \mathrm{~ms})$.
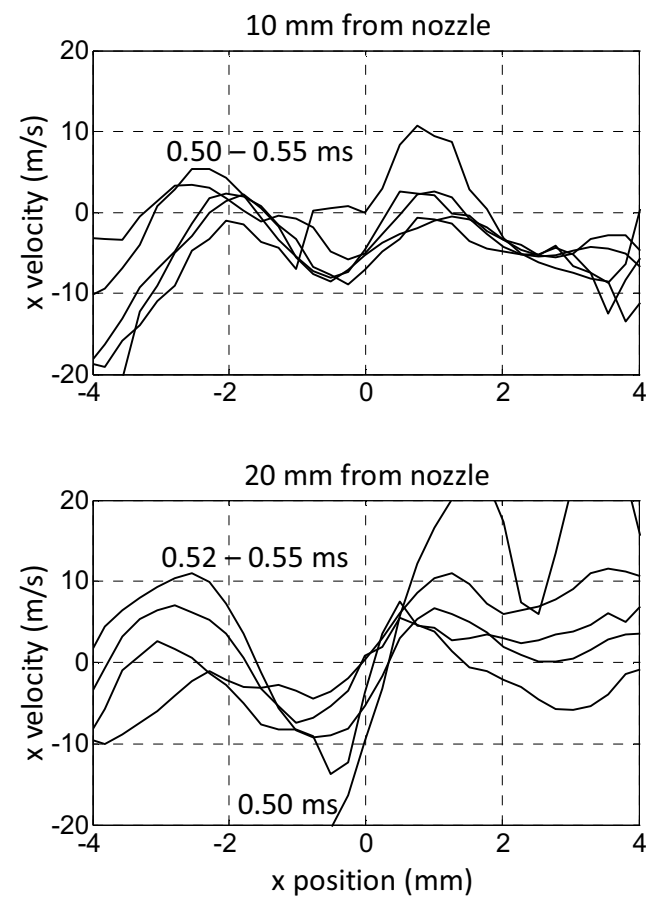

Figure 7. $x$ velocity profiles $(0.50-0.55 \mathrm{~ms})$.

The same effect is recorded also $20 \mathrm{~mm}$ form the nozzle with some delay. At this location the velocity is reduced gradually to a minimum of $\sim 50 \mathrm{~m} / \mathrm{s}$ in the time span $0.58-0.75 \mathrm{~ms}$. Subsequently the velocity increases again to stabilize at $\sim 80 \mathrm{~m} / \mathrm{s}$ from $1.0 \mathrm{~ms}$ until end of injection. At this location the fluctuations in time are relatively larger compared with the velocity profiles relative to $10 \mathrm{~mm}$. Large fluctuations are also recorded in the $\mathrm{x}$ velocity component but the characteristic shape is still consistent almost at each recorded time.
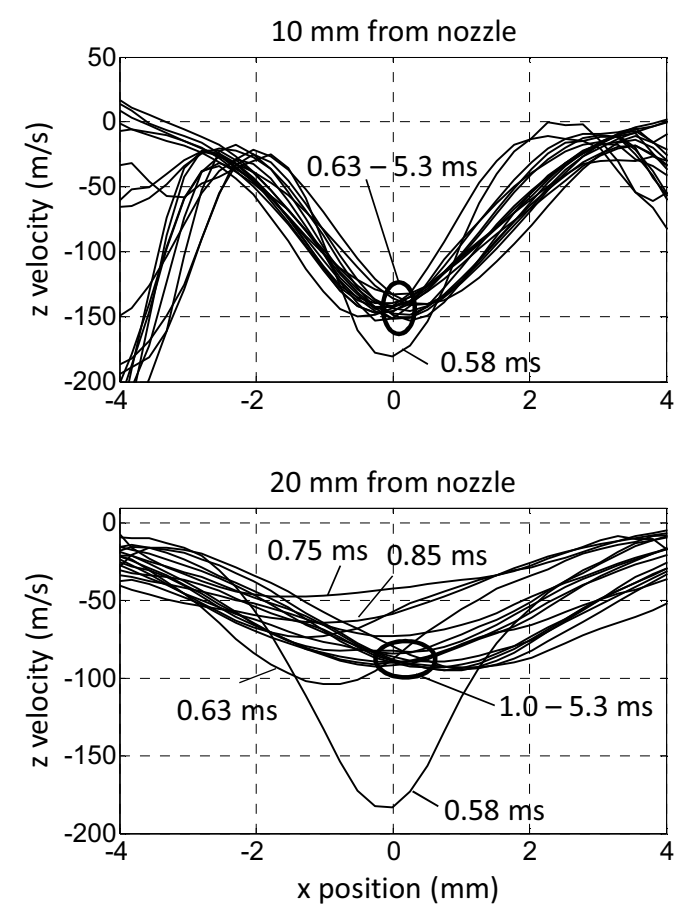

Figure 8. $\mathrm{z}$ velocity profiles $(0.58-5.3 \mathrm{~ms})$.
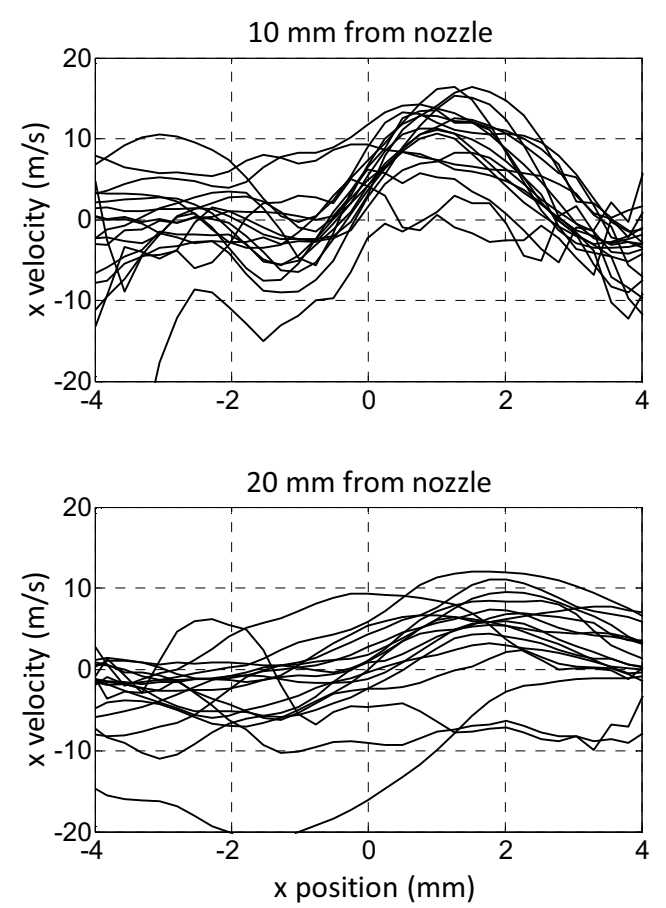

Figure 9. $\mathrm{x}$ velocity profiles $(0.58-5.3 \mathrm{~ms})$.

\subsection{Turbulence}

The accuracy of the PIV measurements is not high enough to derive reliable information about the turbulence quantities in the jet. Other techniques, such as HWA (Hot Wire Anemometer) or LDA (Laser Doppler Anemometer) would have been more suitable. The task is further complicated by the transient nature of the flow. Nevertheless, it is attempted to derive some valuable information about turbulence from the measurements. 
This is done following the scope of this work is to provide as much information as possible to serve as input to CFD simulations. In figure 10 are reported three samples of the instantaneous velocity magnitude at different locations and times. From these profiles it might be possible to make some rough consideration about the turbulence length scales. The average velocity magnitude and the velocity fluctuation RMS is calculated over 100 samples. The turbulence intensity is calculated as the ratio between the average fluctuation (RMS) and the average velocity for each $\mathrm{x}$ position. The measuring system is set to have the highest accuracy at the jet core (i.e. at the location of highest velocity). In this region the values for the turbulence intensity are believed to be somewhat truthful.
$0.52 \mathrm{~ms}$

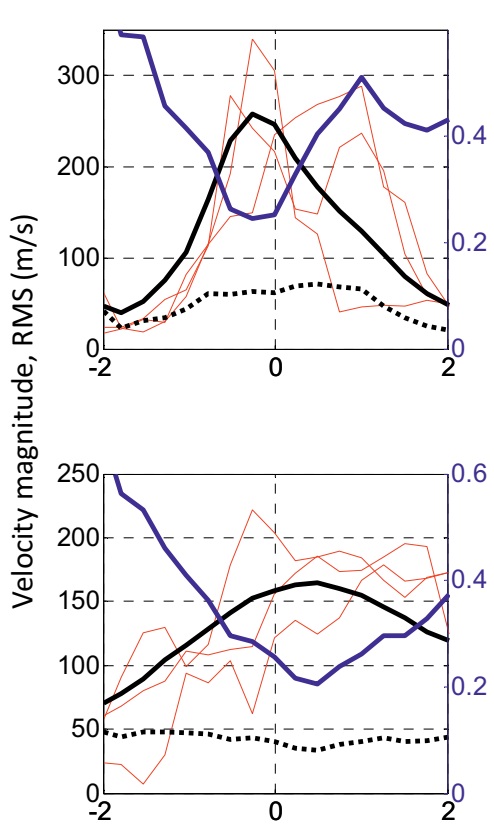

$0.67 \mathrm{~ms}$ $10 \mathrm{~mm}$ from nozzle

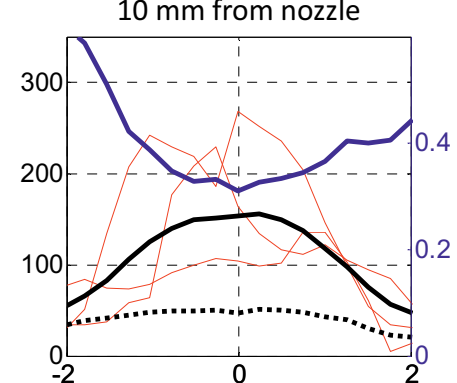

$20 \mathrm{~mm}$ from nozzle

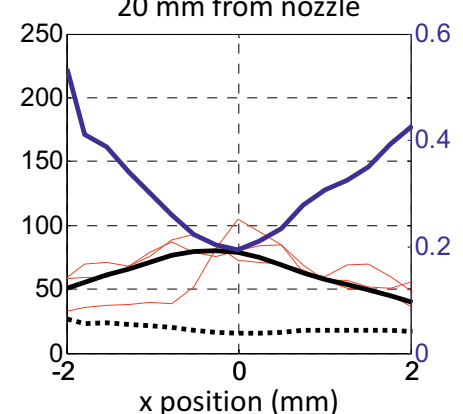

$2.0 \mathrm{~ms}$
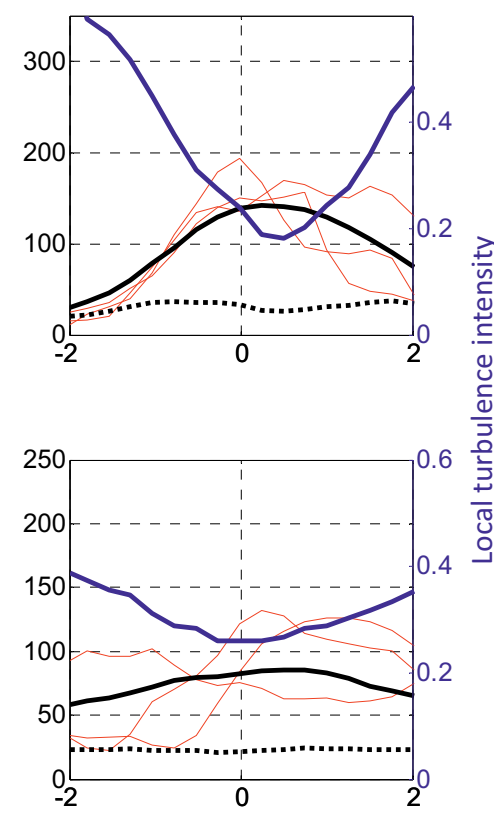

Figure 10. Black: Average velocity magnitude and RMS (average over 100 samples). Blue: turbulence intensity (y axis on the right). Red: three samples of instantenous velocity magnitude.

\section{Thermocouples}

The target is equipped with eight surface thermocouples placed on the impingement area (figure 2). The thermocouple construction and characteristics can be found in [19]. The sensing element is the junction between the two thermocouples parts, this is realized with a thin layer of vaporized metal deposited under vacuum. The result is a device with very low thermal inertia capable to respond to temperature changes of the surrounding within $\mu$ s, hence well capable to resolve the jet timescale $(5 \mathrm{~ms})$. Six thermocouples are placed $5 \mathrm{~mm}$ apart in a row, thus able to resolve the radial effect of the jet. Further, two thermocouples are placed at $\mathrm{R}=25 \mathrm{~mm}$ providing information about the axial-symmetry of the jet.

The results for the thermocouple experiment are reported in figure 11. The plots are based on the average of 50 samples. Measurements upwards and downwards are not carried out simultaneously, but by changing the target inclination. The graphs show the temperature evolution as recorded by the thermocouples. Time 0 is the open signal sent to the injector. A measureable thermal interaction with the target begins with $\sim 2 \mathrm{~ms}$ delay. In the results it is possible to follow the evolution of the wall jet in different directions with the thermocouples located at different radii. In all plots it is possible to appreciate the time it takes for the wall jet to travel away from the impingement point. Notably, for $60^{\circ}$ the jet never reaches the thermocouple at $\mathrm{R}=20 \mathrm{~mm}$ in the upwards direction. The highest thermal interaction is measured for the case $0^{\circ}$ at $\mathrm{R}=0 \mathrm{~mm}$ with a temperature change of $\sim 0.75^{\circ} \mathrm{C}$ in $\sim 4 \mathrm{~ms}$. The location of the strongest thermal interaction for the other cases shifts upwards. This is most clear for case $60^{\circ}$ where the lowest temperature is recorded at $\mathrm{R}=$ $5 \mathrm{~mm}$ instead that $\mathrm{R}=0 \mathrm{~mm}$.

In some of the curves it is possible to notice consistent fluctuations which are likely due to electromagnetic noise (e.g. $\mathrm{R}=10 \mathrm{~mm}$ at $\sim 5 \mathrm{~ms}$, any plot). Statistical tools reveal a very high repeatability of the results with a normalized standard deviation below $1 \%$. This suggests that the target thermal inertia plays a significant role in dampening the effect of the turbulent fluctuations on the surface temperature. The quality of the results is also comforted by the accuracy of the trends in both space and time as discussed in [13]. 


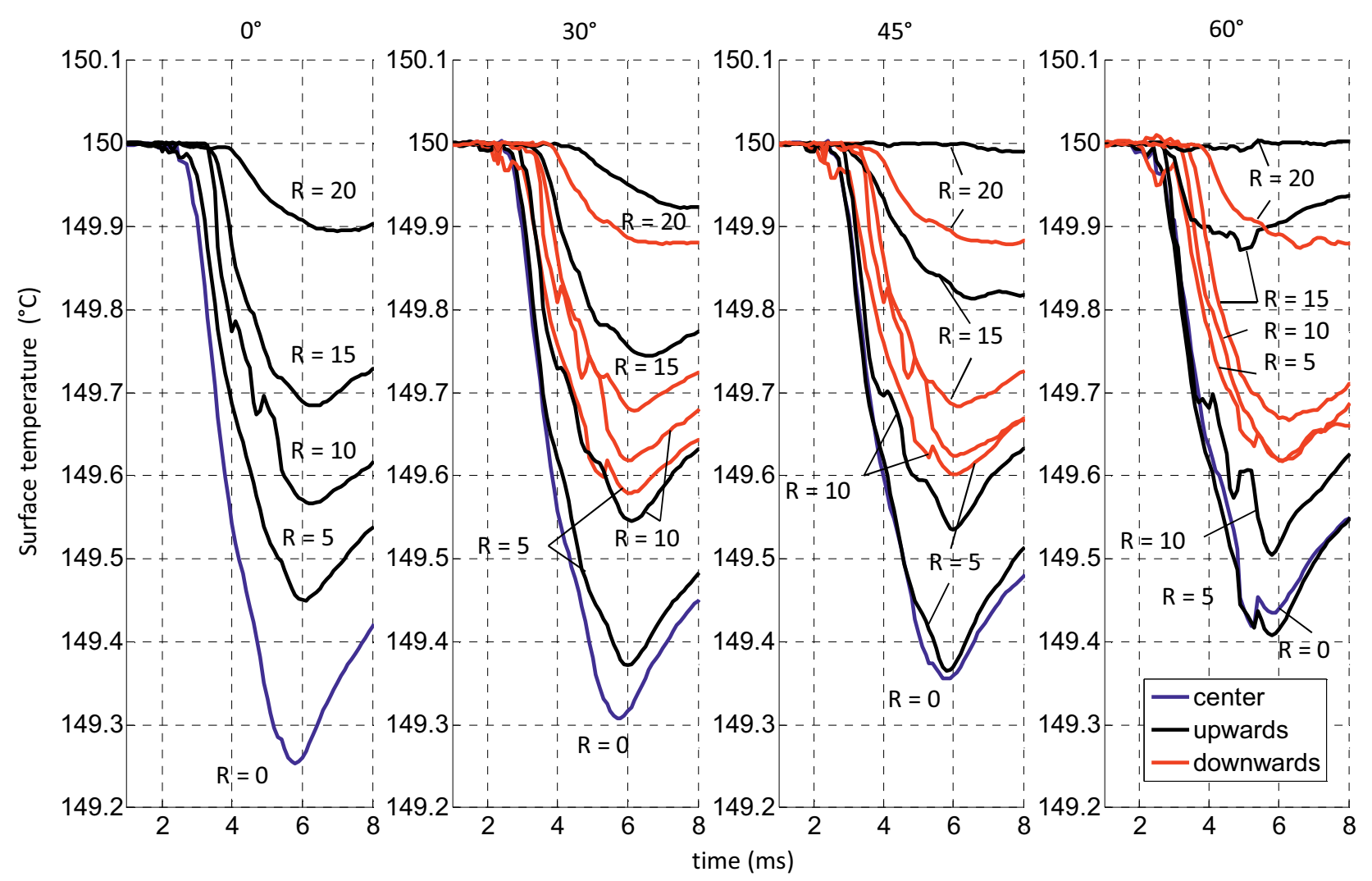

Figure 11. Thermocouple results for different target inclinations. Parameterized by the distance from the target centre in different radial directions $(\mathrm{R}$ in $\mathrm{mm})$.

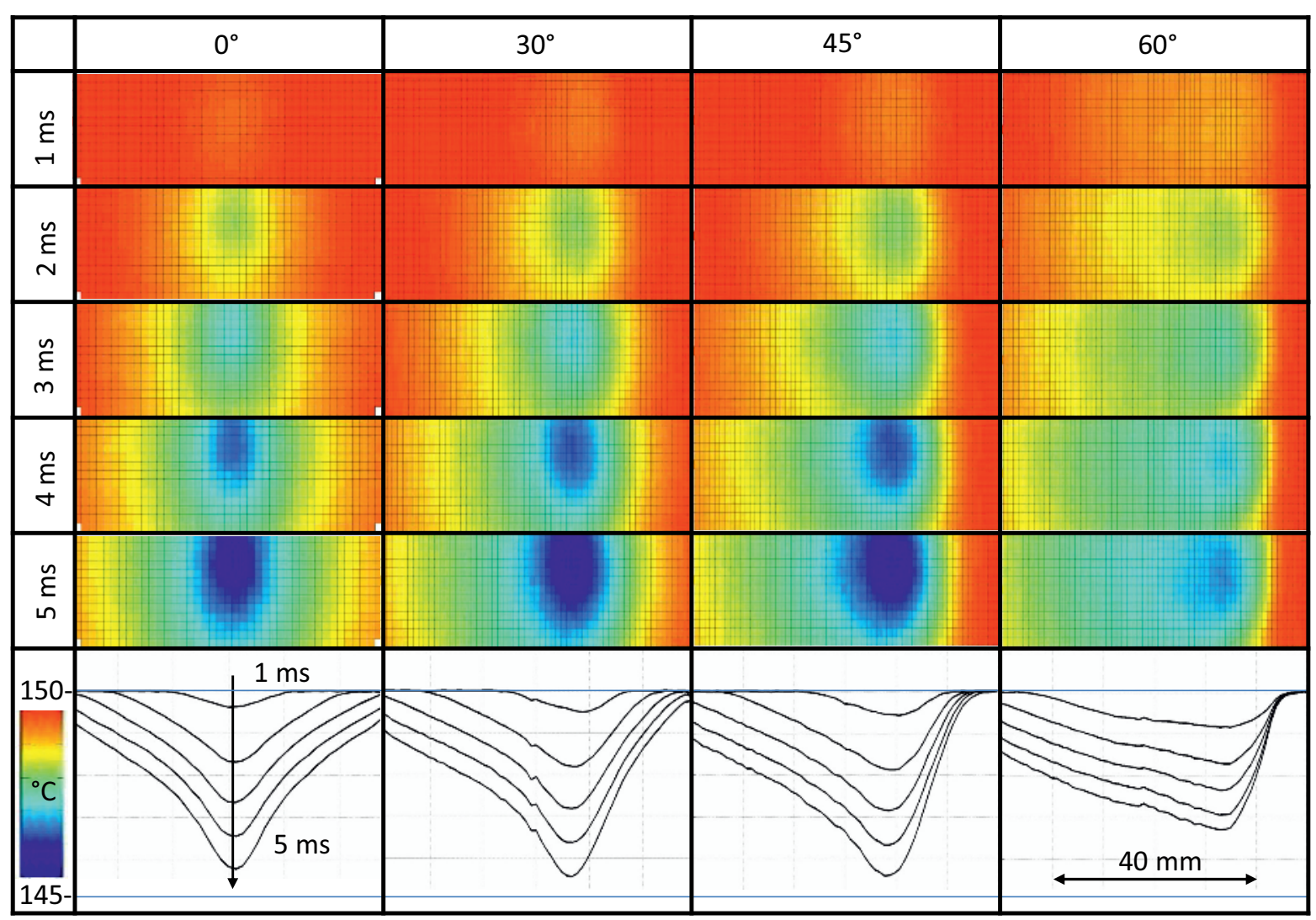

Figure 12. Thermal camera results.

$\overline{\text { a Corresponding bovo@chalmers.se }}$ 


\section{Thermal camera}

Further an experiment is carried out using a thermal camera to measure the target temperature evolution in space and time. The setup is carried out closely following the guidelines presented in [21]. The thermal camera is arranged to sample a strip of the target along the line of maximum inclination. The sampling rate corresponds to an integration time of $0.5 \mathrm{~ms}$, thus the jet $(5 \mathrm{~ms})$ is resolved with 10 frames. The accuracy in the temperature range of interest is $\sim 0.1{ }^{\circ} \mathrm{C}$. The camera frame is $64 \mathrm{x} 12$ pixels, which results in a spatial resolution of $\sim 1 \mathrm{x} 1 \mathrm{~mm}$.

A calibration device is purposely designed to provide a flat surface with uniform controlled temperature. The surface is prepared with high emissivity coating NEXTEL Velvet Coating 811-21. The paint characteristics are reported in [21]. The target surface is painted using the same coating with a $\sim 50 \mu \mathrm{m}$ thickness. This layer is thick enough to have a significant thermal insulation effects, these effects are discussed in the following section.

Figure 12 presents a collection of the results. All plots and figures are based on the average of 20 samples. The top part the table displays the surface temperature evolution in using color contour plot. In the bottom the same results are presented in graphs, in this case the values are taken along the line of highest thermal interaction (i.e. lowest temperature). These results can be directly used for comparison with CFD simulations. A physical interpretation of the results is carried out by the authors and presented in [13].

\section{Discrepancy between surface temperature measurement techniques}

The transient thermal response of the target is significantly different whether its surface is untreated (polished aluminum as in the thermocouples experiment) or coated with high emissivity paint (thermal camera experiment). This is clearly noticeable comparing the magnitude of the measurements obtained with the different methods. The thermal effects of the coating can be estimated performing the semi-infinite body analysis [22]. The graphs in figure 13 indicate the temperature evolution at the surface and $0.1 \mathrm{~mm}$ into the material. These correspond to a target entirely made of aluminum or paint respectively. The target at a homogeneous temperature of $150{ }^{\circ} \mathrm{C}$ is suddenly exposed to a medium at $20{ }^{\circ} \mathrm{C}$ with a heat transfer coefficient of $1000 \mathrm{~W} \mathrm{~m}^{-2} \mathrm{~K}^{-1}$ (estimated value in the stagnation region from $\mathrm{CFD}$ analysis). The results plotted in figure 13 show the significant transient thermal effect of the coating.
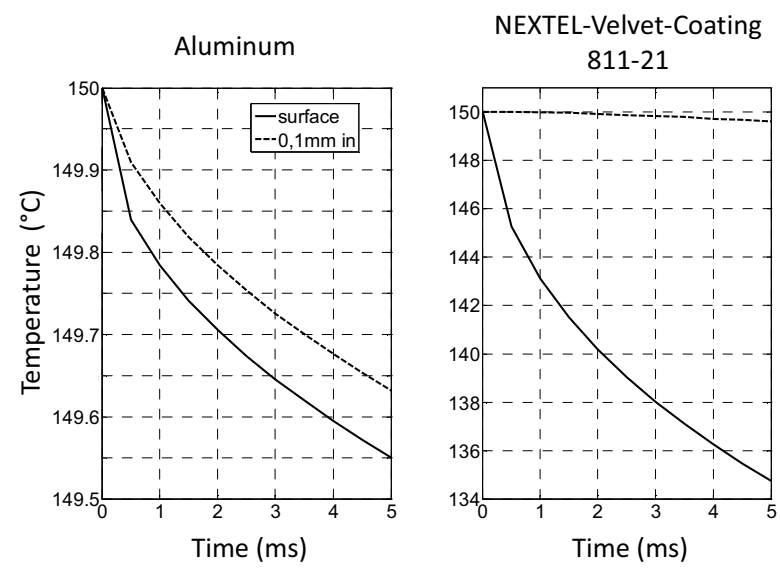

Figure 13. Target temperature evolution. Left: polished aluminum. Right: high emissivity black coating.

\section{Conclusions}

A well defined single pulse impinging jet is measured with different techniques. The main purpose is to collect information on the the velocity field and fluid-solid thermal interaction. The combination of these results gives the complete definition of the boundary conditions necessary to setup a CFD simulation. More specifically, the geometry and physical conditions are reported with enough information to reproduce the case with a numerical simulation. The jet velocity profile is resolved in space and time at two location suitable to serve as inlet velocity boundary condition. The fluid-solid thermal interaction can be directly compared with the temperature evolution of the target surface. This is measured with two different techniques. Thermocouples with high time resolution and thermal camera with high spatial resolution. The results are presented for four cases, i.e. with the target inclined at $0^{\circ}, 30^{\circ}, 45^{\circ}$ and $60^{\circ}$ relative to the jet axis.

Cross interpretation of the results presented in this paper can be used to study pulse jets and their thermal effects at impingement. Moreover, interpretation of the findings can be reinforced by CFD studies. One such analysis by the same authors of this work is reported in [13].

\section{References}

1. K. Jambunethan, E. Lai, M. A. Moss and B. L. Button, Int. J. Heat and Fluid Flow, Vol. 13, No. 2 106-115 (1992)

2. R. Viskanta, Experimental Thermal and Fluid Science 6:111-134 (1993)

3. J. W. Baughn, and S. Shimizu, ASME J. Heat Transfer 111/1097 (1989)

4. D. Cooper, D. C. Jackson, B. E. Launder, G. X. Liao, Int. J. Heat Mass Transf. 36, 2675-2684 (1993)

5. V. Katti, S.V. Prabhu, Int. J. Heat Mass Transfer, Vol. 51 4480-4495 (2008)

6. D. Lytle, B.W. Webb, Int. J. Heat Mass Transfer 37 1687-1697 (1994)

\footnotetext{
$\overline{{ }^{a} \text { Corresponding bovo@chalmers.se }}$
} 
7. N. Gao, H. Sun, D. Ewing, Int. J. Heat Mass Transfer 46 2557-2569 (2003)

8. T. S. O'Donovan, D. B. Murray, Int. J. Heat and Mass Transfer 50 3291-3301 (2007)

9. T. S. O'Donovan, D. B. Murray, Int. J. Heat and Mass Transfer 50 3302-3314 (2007)

10. N. Gao, D. Ewing, Int. J. of Heat and Fluid Flow 27 33-41 (2006)

11. M.F. Koseoglu, S. Baskaya, Int. J. of Thermal Sciences Vol. 47 1332-1346 (2008)

12. T. Husberg, S. Gjiria, I. Denbratt, Piston Temperature Measurements by use of thermographic phosphors and thermocouples in a heavy-duty diesel engine run under partially premixed conditions, SAE 2005-01-1646 (2005)

13. M. Bovo, B. Rojo, Single Pulse Jet Impingement on Inclined Surface. Heat Transfer and Flow Field, SAE 13ICE-0063 (2013)

14. L. F. A. Azevedo, B. W. Webb M. Queiroz, Experimental Thermal and Fluid Science, 8:206-213 (1994)

15. T. Janetzke, W. Nitsche, International Journal of Heat and Fluid Flow 30 877-885 (2009)

16. P. Xu, B. Yu, S. Qiu, H. J. Poh, A. S. Mujumdar, International Journal of Thermal Sciences 49 1247$1252(2010)$

17. H. M. Hofmann, D. L. Movileanu, M. Kind, H. Martin, International Journal of Heat and Mass Transfer 50 3638-3648 (2007)

18. R. Baert, A. Klaassen, Direct Injection of High Pressure Gas: Scaling Properties of Pulsed Turbulent Jets, SAE 2010-01-2253 (2010)

19. Product Information Coaxial Surface Thermocouple Probes, Medtherm Corporation, Alabama USA

20. M. Raffel, C. Willert, J. Kompenhans, Particle Image Velocimetry, A Practical Guide. Springer, p. 253 (1998)

21. C. Arroyo Osso, Aerothermal Inestigation of an Intermediate Duct, $\mathrm{PhD}$ Thesis, Chalmers University of Technology (2009)

22. Y. A. Cengel, Heat transfer a practical approach second edition, Mc Graw-Hill (2003) 